\title{
Combined immune checkpoint blockade for metastatic uveal melanoma: a retrospective, multi-center study
}

\author{
Markus V. Heppt ${ }^{1,2}$, Teresa Amaral ${ }^{3,4}$, Katharina C. Kähler ${ }^{5}$, Lucie Heinzerling ${ }^{2}$, Jessica C. Hassel ${ }^{6}$, Markus Meissner ${ }^{7}$, \\ Nicole Kreuzberg ${ }^{8}$, Carmen Loquai $^{9}$, Lydia Reinhardt ${ }^{10}$, Jochen Utikal ${ }^{11}$, Evelyn Dabrowski ${ }^{12}$, Anja Gesierich $^{13}$, \\ Claudia Pföhler ${ }^{14}$, Patrick Terheyden ${ }^{15}$, Kai-Martin Thoms ${ }^{16}$, Lisa Zimmer ${ }^{17}$, Thomas K. Eigentler ${ }^{3}$, \\ Michael C. Kirchberger ${ }^{2}$, Henner M. Stege ${ }^{9}$, Friedegund Meier ${ }^{10}$, Max Schlaak ${ }^{1}$ and Carola Berking ${ }^{1,2^{*}}$
}

\begin{abstract}
Background: Uveal melanoma (UM) is highly refractory to treatment with dismal prognosis in advanced stages. The value of the combined checkpoint blockade with CTLA-4 and PD-1 inhibition in metastatic UM is currently unclear.

Methods: Patients with metastatic or unresectable UM treated with ipilimumab in combination with a PD-1 inhibitor were collected from 16 German skin cancer centers. Patient records of 64 cases were analyzed for response, progression-free survival (PFS), overall survival (OS), and safety. Clinical parameters and serum biomarkers associated with OS and treatment response were determined with Cox regression modelling and logistic regression.
\end{abstract}

Results: The best overall response rate to combined checkpoint blockade was $15.6 \%$ with 3.1 and $12.5 \%$ complete and partial response, respectively. The median duration of response was 25.5 months (range 9.0-65.0). Stable disease was achieved in $21.9 \%$, resulting in a disease control rate of $37.5 \%$ with a median duration of the clinical benefit of 28.0 months (range 7.0-65.0). The median PFS was 3.0 months ( $95 \%$ Cl 2.4-3.6). The median OS was estimated to 16.1 months (95\% Cl 12.9-19.3). Regarding safety, 39.1\% of treated patients experienced a severe, treatment-related adverse event according to the CTCAE criteria (grade 3: 37.5\%; grade 4: 1.6\%). The most common toxicities were colitis (20.3\%), hepatitis (20.3\%), thyreoiditis (15.6\%), and hypophysitis (7.8\%). A poor ECOG performance status was an independent risk factor for decreased OS ( $p=0.007)$.

Conclusions: The tolerability of the combined checkpoint blockade in UM may possibly be better than in trials on cutaneous melanoma. This study implies that combined checkpoint blockade represents the hitherto most effective treatment option available for metastatic UM available outside of clinical trials.

Keywords: Ipilimumab, Nivolumab, Combined immune checkpoint blockade, Uveal melanoma, Biomarker

\footnotetext{
* Correspondence: Carola.Berking@uk-erlangen.de

'Department of Dermatology and Allergy, Munich University Hospital (LMU),

Frauenlobstr. 9-11, 80337 Munich, Germany

${ }^{2}$ Department of Dermatology, University Hospital Erlangen,

Friedrich-Alexander-University Erlangen-Nürnberg (FAU), Ulmenweg 18,

91054 Erlangen, Germany

Full list of author information is available at the end of the article
}

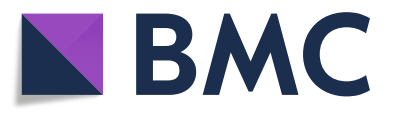

(c) The Author(s). 2019 Open Access This article is distributed under the terms of the Creative Commons Attribution 4.0 International License (http://creativecommons.org/licenses/by/4.0/), which permits unrestricted use, distribution, and reproduction in any medium, provided you give appropriate credit to the original author(s) and the source, provide a link to the Creative Commons license, and indicate if changes were made. The Creative Commons Public Domain Dedication waiver (http://creativecommons.org/publicdomain/zero/1.0/) applies to the data made available in this article, unless otherwise stated. 


\section{Background}

Uveal melanoma (UM) is a malignant tumor of the eye that originates from the pigment cells of the choroid layer or the ciliary body which is clinically and biologically distinct from cutaneous melanoma. Although the incidence is much lower than that of cutaneous melanoma, UM belongs to the most common malignant intraocular tumors in adults [1]. In approximately 50\% of all cases, patients develop distant metastasis during the course of the disease, which affects predominantly the liver. Clinical risk factors for metastases are posterior localization in the eye, tumor size of more than $10 \mathrm{~mm}$, and presence of vascular loops. Molecular biomarkers associated with a higher risk of metastasis are monosomy 3 or genomic alterations of BAP-1 [2]. Once distant metastases have occurred, the prognosis is dismal with an average survival time of approximately 1 year across all therapeutic regimens [3].

Patients with metastatic UM have so far benefited little or not at all from the treatment innovations achieved in cutaneous melanoma in recent years. Neither targeted therapy with MEK inhibitors nor checkpoint blockade with ipilimumab or PD-1 inhibitors as monotherapy was able to significantly improve the prognosis of patients with UM $[4,5]$. The response rates were consistently in the single-digit percentage range in a panel of previous studies [6-9]. In cutaneous melanoma, combined checkpoint blockade with ipilimumab and nivolumab revealed response rates and survival outcomes superior to PD-1 inhibitor monotherapy, albeit at the cost of high immune-related toxicity [10]. However, the significance of combined checkpoint blockade in UM is unclear and has only been investigated in case reports and small case series $[6,11,12]$. In this study, we evaluate the clinical course of 64 patients with metastatic UM who received combined checkpoint blockade. We report clinical outcomes with respect to response, survival, and adverse events (AE). Furthermore, clinical and laboratory parameters were investigated which may have prognostic value in UM patients treated with checkpoint blockade.

\section{Patients and methods}

\section{Patient population and study approval}

This study was designed as a retrospective multi-center explorative analysis. Patients were included if they had a diagnosis of stage IV UM and received combined checkpoint blockade of ipilimumab with a PD-1 inhibitor in any treatment line. A follow-up period of at least 3 months was required. The clinical data of 64 patients from 16 German skin cancer centers who met the inclusion criteria were investigated. The cases were collected from June 23, 2018 to October 4, 2019. Clinical data and the treatment outcomes of interest were extracted from the original patient records and merged into a central database prior to analysis. This study was approved by the institutional review board of the medical faculty of the Munich University Hospital (approval number 41316 UE) and was conducted in accordance with the principles of the Helsinki Declaration in its current version.

\section{Data collection and treatment outcomes}

The clinical data recorded at baseline prior to immunotherapy comprised demographics with Eastern Cooperative Oncology Group (ECOG) performance status, available information on the genotype, sites of metastasis, number of organ systems affected by metastases, and previous antineoplastic therapies. As potential serum biomarkers, lactate dehydrogenase (LDH), C-reactive protein (CRP), and the relative counts of lymphocytes (RLC), neutrophils (RNC), and eosinophils (REC) were specifically collected from patient charts and analyzed for their prognostic value $[13,14]$.

Combined checkpoint blockade was carried out using different treatment schedules (Table 1). Ipilimumab was given at either $3 \mathrm{mg} / \mathrm{kg}$ or $1 \mathrm{mg} / \mathrm{kg}$ body weight for up to 4 treatment cycles. Nivolumab was applied at $1 \mathrm{mg} / \mathrm{kg}$ together with ipilimumab, followed by $3 \mathrm{mg} / \mathrm{kg}$ every 2 weeks $(\mathrm{Q} 2 \mathrm{~W})$ as maintenance therapy. Treatment with pembrolizumab was applied every 3 weeks (Q3W) at 2 $\mathrm{mg} / \mathrm{kg}$. Patients were treated until disease progression or until the development of unacceptable toxicity. AE were retrospectively graded by the site investigators based on the patient records and clinical outcomes according to the Common Terminology Criteria for Adverse Events (CTCAE) v5.0 published by the National Institutes of Health in 2017. Immune-related adverse events were managed according to pertinent guidelines and algorithms that were previously published $[15,16]$. Besides, fatal adverse events and events leading to permanent discontinuation of treatment were specifically recorded and evaluated. The best radiologic response to treatment was assessed by the site investigators and indicated as complete response, partial response, stable disease, or progressive disease based on the RECIST criteria version 1.1 [17]. Complete response and partial response were summarized as best overall response rate (ORR). Complete response, partial response, and stable disease were summarized as disease control rate (DCR).

\section{Statistical analyses}

Overall survival (OS) and progression-free survival (PFS) were calculated as the time from the initiation of the first cycle of combined checkpoint blockade until melanomaspecific or treatment-related death and disease progression, respectively. Time-to-event analyses were calculated where death or progression were considered as events. If neither occurred or if patients were lost to follow-up, the date of the last documented presentation was used as a 
Table 1 Baseline characteristics of the patient population

\begin{tabular}{|c|c|}
\hline & $\begin{array}{l}\text { Patient population } \\
n=64(100 \%)\end{array}$ \\
\hline \multicolumn{2}{|l|}{ Gender } \\
\hline Male & $33(51.6)$ \\
\hline Female & $31(48.4)$ \\
\hline \multicolumn{2}{|l|}{ Age } \\
\hline$<60$ years & $28(43.8)$ \\
\hline$\geq 60$ years & $36(56.2)$ \\
\hline \multicolumn{2}{|l|}{ GNAQ } \\
\hline Mutated & $8(12.5)$ \\
\hline Wildtype & $8(12.5)$ \\
\hline Unknown & $48(75.0)$ \\
\hline \multicolumn{2}{|l|}{ GNA11 } \\
\hline Mutated & $10(15.6)$ \\
\hline Wildtype & $5(7.8)$ \\
\hline Unknown & $49(76.6)$ \\
\hline \multicolumn{2}{|l|}{ ECOG status } \\
\hline 0 & $49(76.6)$ \\
\hline 1 & $11(17.2)$ \\
\hline 2 & $1(1.6)$ \\
\hline 3 & $1(1.6)$ \\
\hline Unknown & $2(3.1)$ \\
\hline \multicolumn{2}{|l|}{ Serum LDH } \\
\hline Normal & $28(43.8)$ \\
\hline Elevated (>ULN) & $33(51.6)$ \\
\hline Unknown & $3(4.7)$ \\
\hline \multicolumn{2}{|l|}{ Previous systemic therapies } \\
\hline 0 & $50(78.1)$ \\
\hline 1 & $12(18.8)$ \\
\hline$\geq 2$ & $2(3.1)$ \\
\hline Previous ipilimumab monotherapy & $2(3.1)$ \\
\hline Previous PD-1 inhibitor monotherapy & $12(18.8)$ \\
\hline \multicolumn{2}{|l|}{ Liver-directed therapies } \\
\hline 0 & $33(51.6)$ \\
\hline 1 & $30(46.9)$ \\
\hline$\geq 2$ & $1(1.6)$ \\
\hline \multicolumn{2}{|l|}{ Metastatic sites $^{a}$} \\
\hline Liver & $58(90.6)$ \\
\hline Lung & $23(35.9)$ \\
\hline Bone & $17(26.6)$ \\
\hline Lymph nodes & $12(18.8)$ \\
\hline CNS & $4(6.3)$ \\
\hline \multicolumn{2}{|l|}{ Treatment regimen } \\
\hline Ipilimumab $3 \mathrm{mg} / \mathrm{kg}$ + nivolumab $1 \mathrm{mg} / \mathrm{kg}$ Q3W, followed by nivolumab $3 \mathrm{mg} / \mathrm{kg}$ Q2W & $59(92.2 \%)$ \\
\hline Ipilimumab $1 \mathrm{mg} / \mathrm{kg}$ + pembrolizumab $2 \mathrm{mg} / \mathrm{kg}$ Q3W, followed by pembrolizumab 2 mg/kg Q3W & $5(7.8 \%)$ \\
\hline
\end{tabular}


censored observation. The survival and progression probabilities were indicated with the Kaplan-Meier method for censored failure time data assuming proportional hazards. The survival curves were compared with the log-rank test [6]. The duration of the clinical response and clinical benefit was defined as time from treatment initiation to progressive disease if a response or stable disease was achieved, respectively. The time to response was defined as time from treatment start until a response was evident radiologically.

Cox proportional hazards regression modelling was applied to investigate the relationship of clinical risk factors and serum biomarkers with OS. Cox regression was performed as a univariate and multivariate analysis in a stepwise approach [6]. Imputation of missing data was not allowed and patients with missing values of a given parameter were excluded from the analysis. Hazard ratios (HR) with 95\% confidence intervals (CI) were calculated to quantify the impact on survival. $P$-values were calculated based on Wald statistics [6]. The association of treatment response as a categorical variable with clinical characteristics or serum biomarkers was investigated with the Chi-square test and logistic regression, as appropriate. In all cases, two-tailed $p$-values were calculated and considered significant with values $p<0.05$. All analyses were carried out with SPSS statistics version 23.0 (IBM) or GraphPad Prism version 5.01 (GraphPad Software).

\section{Results}

A total of $64(100 \%)$ patients with metastatic UM were included. Fifty patients (78.1\%) were naïve to systemic treatment and received combined checkpoint blockade as first-line systemic therapy. Regarding genotype, the presence of monosomy 3 as risk factor was specifically investigated in 7 patients and identified in 2 of them. BRAF, NRAS and KIT were analyzed and reportedly wildtype as expected in 30, 22, and 20 patients, respectively. Mutations and inactivations of MBD4 which were previously linked to a hypermutator profile with high sensitivity to PD-1 inhibition were not investigated in any case $[18,19]$.

Previous ipilimumab and PD-1 inhibitor monotherapy were applied in $2(3.1 \%)$ and $12(18.8 \%)$ cases, respectively. Both patients treated with ipilimumab before showed PD. Specifically, 4 patients $(6.3 \%)$ had received nivolumab and 8 (12.5\%) pembrolizumab before. In 4 cases, SD was achieved while 8 patients showed PD upon PD-1 inhibitor monotherapy. The median duration of the clinical benefit was 6.5 months in the 4 patients with SD. Liver-directed therapies were reported in 31 patients (48.4\%). Most patients had an ECOG status of 0 $(n=49,76.6 \%)$. Serum LDH was elevated in 33 cases $(51.6 \%)$ at baseline. Other baseline characteristics are listed in detail in Table 1. Ipilimumab plus nivolumab was given in 59 patients $(92.2 \%)$, while 5 patients $(7.8 \%)$ received ipilimumab plus pembrolizumab. The median number of treatment cycles was 3 (range 1-4) for the combination of ipilimumab with a PD-1 inhibitor in the induction phase, and 0 (range 0-27) for PD-1 inhibitor maintenance therapy in the overall population. A total of 19 patients (29.7\%) received a PD-1 inhibitor maintenance therapy. Among these, the median number of PD-1 inhibitor cycles was 3 (range 1-27).

The best ORR to combined checkpoint blockade was $15.6 \%(n=10)$ relating to the entire population (4 patients were not evaluable for a radiologic response). Two patients achieved a complete response (3.1\%) and 8 (12.5\%) a partial response. The median duration of response was 25.5 months (range 9.0-65.0). Stable disease was achieved in further 14 cases $(21.9 \%)$, resulting in a disease control rate of $37.5 \%$ with a median duration of the clinical benefit of 28.0 months (range 7.0-65.0) (Table 2). The median PFS was 3.0 months (95\% CI 2.4-3.6). The median OS was estimated to 16.1 months (95\% CI 12.9-19.3) with a median follow-up period of 9.2 months (95\% CI 7.8-10.6) (Fig. 1).

The median time to response in patients with $\mathrm{CR}$ or PR after treatment initiation was 12 weeks (range 5-31). For the patients with SD, the median duration until the benefit was observed also amounted to 12 weeks (range 9-30). Interestingly, all 4 patients with SD after previous single PD-1 inhibitor blockade had PD to combined checkpoint blockade. Among the remaining 8 patients with PD after previous single PD-1 inhibitor blockade, one achieved a PR to combined checkpoint blockade. Thus, these data suggest that the effects of single and combined checkpoint blockade were observed independently from each other.

A total of $78 \mathrm{AE}$ were reported in 39 patients. Thus, the majority of patients developed any treatment-related $\mathrm{AE}(60.9 \%)$. Of all events, $37 \mathrm{AE}$ were graded as severe (grade $3+4)$. They were observed in 25 patients $(39.1 \%$; grade 3: $37.5 \%$; grade $4: 1.6 \%)$. The treatment was discontinued in 25 cases (39.1\%) due to unacceptable toxicity. However, no treatment-related deaths occurred during treatment or the observation period. The most common events were colitis (20.3\%), hepatitis $(20.3 \%)$,

Table 2 Best response rates to combined checkpoint blockade

\begin{tabular}{lll}
\hline & Cases (\%) & $\begin{array}{l}\text { Cumulative } \\
\text { percentage (\%) }\end{array}$ \\
\hline Complete response & $2(3.1)$ & 3.1 \\
Partial response & $8(12.5)$ & $15.6(\mathrm{ORR})$ \\
Stable disease & $14(21.9)$ & $37.5(\mathrm{DCR})$ \\
Progressive disease & $36(56.3)$ & 93.8 \\
Unknown & $4(6.3)$ & 100 \\
Total & $64(100)$ & 100 \\
\hline
\end{tabular}

Abbreviations: ORR Objective response rate, $D C R$ Disease control rate 


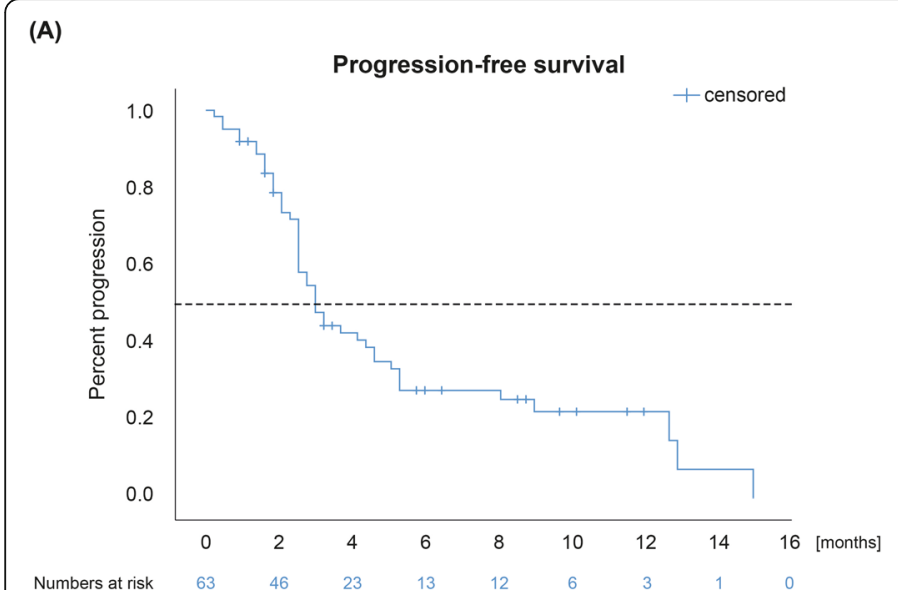

(B)

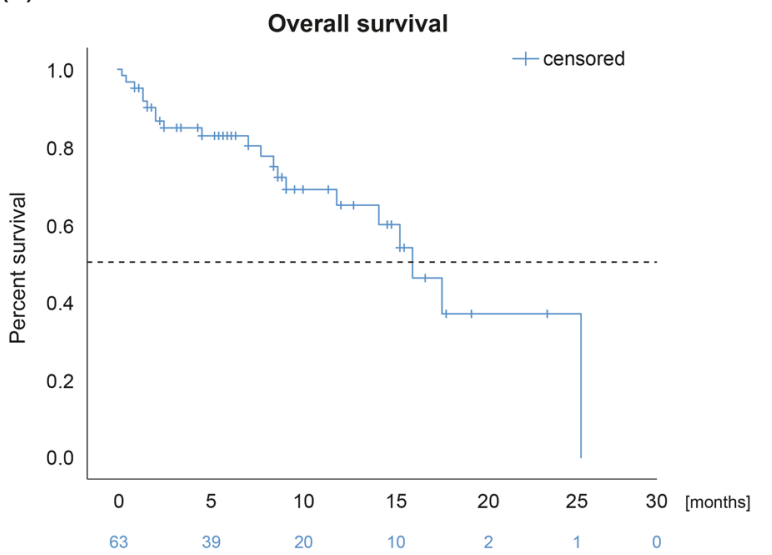

Fig. 1 Kaplan-Meier estimates of the patient population for a progression-free survival (PFS) and $\mathbf{b}$ overall survival (OS). The median PFS and OS was estimated to 3.0 months ( $95 \% \mathrm{Cl}$ 2.4-3.6) and 16.1 months (95\% Cl 12.9-19.3), respectively. One patient was not included in the Kaplan-Meier analysis for PFS and OS due to missing data

thyreoiditis (15.6\%), hypophysitis (7.8\%), fever (4.7\%), and myalgia with myositis (4.7\%). In all 5 cases with hypophysitis, the individual hormone axes including $\mathrm{ACTH}$, cortisol, FSH, LH, TSH, and testosterone were investigated but not specifically graded. In 3 cases, the pituitary gland was enlarged in MRI examinations. All patients received systemic replacement of hydrocortisone. All AE are listed in Additional file 1.

In univariate Cox regression, ECOG status $(p=$ $0.000096)$, the presence of bone metastasis $(p=0.011)$, and the best response to checkpoint blockade $(p=0.002)$ were significantly associated with OS (Additional file 2). The risk factors ECOG status, serum LDH, serum levels of CRP, and presence of bone metastasis were further integrated into a multivariate Cox regression model. Of these factors, a significant association with OS was confirmed for ECOG status $(p=0.007)$ only (Table 3 , Fig. 2a).

We recently identified a prognostic score of the serum biomarkers LDH, CRP, and relative eosinophil count (REC) in a cohort of 94 UM patients receiving PD-1 inhibitors [6]. The score assigns one risk point for each unfavorable factor, i.e., elevated LDH, elevated CRP, and a REC $<1.5 \%$, defining four distinct prognostic groups (low, intermediate, high, and very high risk). Each patient receiving combined checkpoint blockade was assigned to a risk group and the score was validated with Kaplan-Meier estimates. Due to a small sample size, patients with low and intermediate risk were pooled. The risk groups showed significantly different survival probabilities $(p=0.000005)$. The median survival times were superior for the low plus intermediate group (17.7 months, 95\% CI 14.7-20.8) compared to the high (15.4 months, 95\% CI 12.7-18.2) and very high risk group (7.1 months, 95\% CI 0.0-16.2) (Fig. 2b). However, the score neither correlated with the response rate $(p=$
$0.609)$ nor with the DCR $(p=0.446)$, suggesting that it was generally prognostic but not specifically predictive for the response to combined checkpoint blockade.

Subgroup analysis were performed for patients with metastasis to the central nervous system (CNS) at treatment initiation and for the treatment responders. Four patients showed an involvement of the CNS. Two of them had neurological symptoms. Two patients achieved SD, 2 showed PD. The median PFS for the CNS subgroup was 3.0 months (95\% CI 0.0-6.1) while the median OS was not reached. In contrast, none of the treatment responders (CR or PR) had CNS involvement when the treatment was initiated (Table 4). The median time from detection of the primary tumor to metastatic disease was 43 months among the responders. Data on the assessment of the risk of metastasis formation of the primary tumors were sparse, as e.g. the presence of monosomy 3 or the MBD4 status was not investigated in any of the responders.

Table 3 Multivariate Cox regression analysis of clinical parameters and serum biomarkers

\begin{tabular}{llll}
\hline Parameter & Category & HR $(95 \% \mathrm{Cl})$ & $P$-value \\
\hline ECOG status & n.a. (ordinal) & $3.19(1.36-7.47)$ & $0.007^{*}$ \\
LDH & normal & 1 & 0.428 \\
& elevated (>ULN) & $1.83(0.41-8.08)$ & \\
CRP & normal & 1 & 0.534 \\
& elevated (>ULN) & $1.73(0.31-9.74)$ & \\
Bone metastasis & no & 1 & 0.331 \\
& yes & $2.02(0.49-8.27)$ & \\
\hline
\end{tabular}

Four parameters were included in the multivariate Cox regression analysis. Of these factors, ECOG status was significantly associated with overall survival in this model. Abbreviations: Cl Confidence interval, n.a. not applicable, ULN Institutional upper limit of normal, $L D H$ Lactate dehydrogenase, CRP C-reactive protein; ${ }^{*} p<0.05$. 

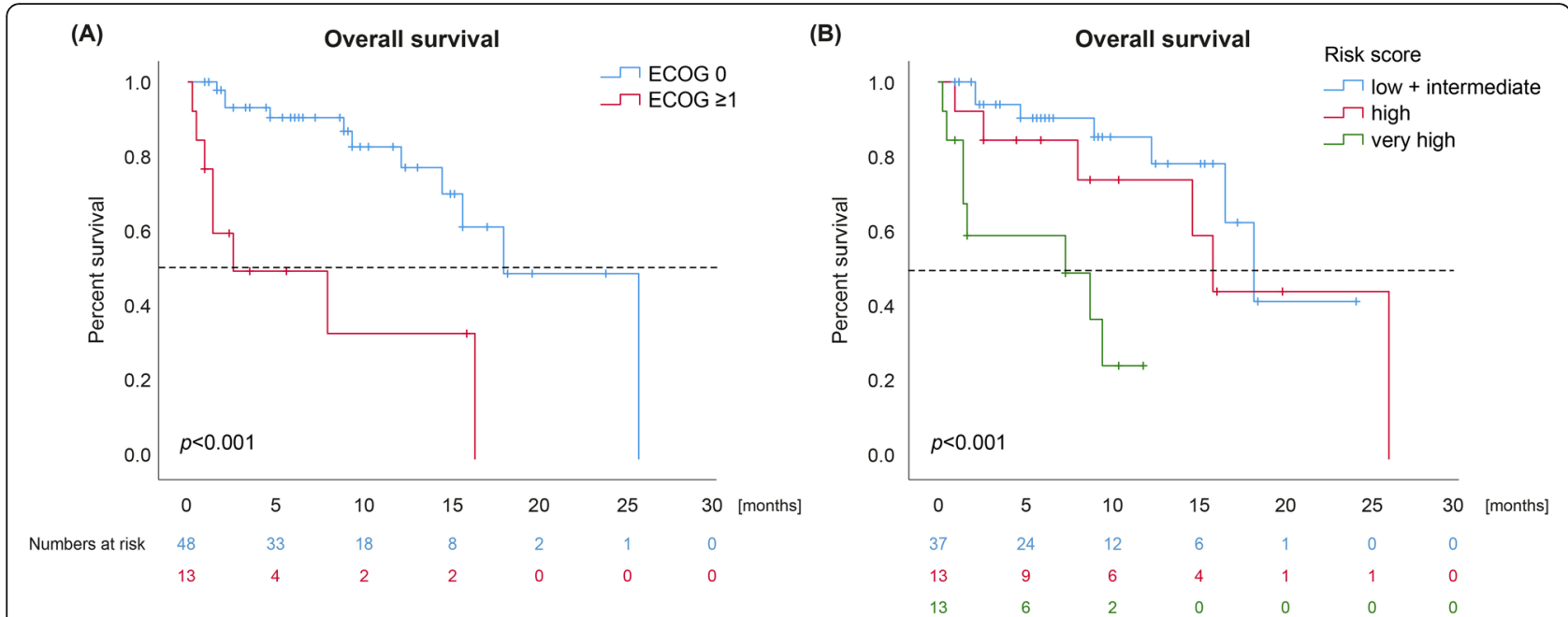

Fig. 2 a Kaplan-Meier estimates for overall survival (OS) according to ECOG performance status. The median OS was 17.7 months (95\% CI 13.122.3) for ECOG 0 versus 2.5 months ( $95 \%$ Cl 0.0-9.6) for ECOG $\geq 1$. Three patients were not included due to missing data. b Kaplan-Meier estimates for OS according to the prognostic score based on the serum parameters LDH, CRP, and REC. The groups with low and intermediate risk were pooled due to a small number of cases. The median OS was 17.7 months (95\% Cl 14.7-20.8) in the low plus intermediate group versus 15.4 months $(95 \% \mathrm{Cl} 12.7-18.2)$ in the high risk group versus 7.1 months ( $95 \% \mathrm{Cl} 0.0-16.2)$ in the very high risk group. The $p$-values indicated were calculated with the log-rank test. One patient was not included due to missing data

\section{Discussion}

Here, we present a comparatively large cohort of patients with metastatic UM who were treated with combined checkpoint blockade. We detected a 15.6\% ORR, with a $3.1 \%$ complete and $12.5 \%$ partial response rate. This response rate is in line with our previous report showing $16 \%$ ORR, although only 12 patients were evaluable for their radiologic response and the follow-up time was short [6]. Another case series was recently published from a single-center experience where 2 out of 8 patients treated with nivolumab and ipilimumab had a partial response [11]. Other preliminary data on the efficacy of the combined checkpoint blockade have been proposed as conference abstracts, but appear preliminary to date. Najjar et al. reported results from a multicenter, retrospective analysis in 66 patients from 11 U.S. centers, revealing an ORR of $13 \%$ and a DCR of $31 \%$ [20]. In addition to these estimates in a real-world setting, prospective trials are currently underway. A preliminary analysis of the Spanish phase II trial GEM1402 (NCT02626962) showed an ORR of $12 \%$ and disease stabilization in $52 \%$ of cases [21]. Another phase II trial is currently ongoing in the U.S. in 30 patients with UM (NCT01585194). A recently presented interim analysis revealed an ORR of $17 \%$ and disease control in 50\% [22]. Thus, we conclude that the ORR of $15.6 \%$ identified in this population is a solid estimate for the efficacy of combined checkpoint blockade in UM and a good indicator of what we can expect from the final analyses of the prospective trials. This regimen appears to be significantly superior compared to the sobering efficacy values observed with ipilimumab and PD-1 inhibitor monotherapy [6-9, 23-26]. Considering the data available so far, we conclude that the increase of ORR of the combined blockade versus PD-1 inhibition alone amounts to approximately $10 \%$. Further evidence for a better efficacy of the combined regimen is supported by the observation of complete responders, albeit to a small extent. This is notable as UM is considered a "cold" tumor due to a low mutational burden and a unique immunosuppressive tumor microenvironment [27-29]. Further research is urgently needed to identify the radiologic, immunologic, and molecular determinants for treatment response in this small subset of patients. Regarding safety, the rate of severe $\mathrm{AE}$ was lower compared to the events reported in the pivotal trial in cutaneous melanoma (CheckMate-067) [30]. In particular, the occurrence of potentially life-threatening grade $4 \mathrm{AE}$ was surprisingly low, suggesting that the regimen may be better tolerated in UM. However, it is also conceivable that the retrospective design and the small number of cases of this study causes an underreporting of AE.

Among clinical parameters and serum biomarkers, only the ECOG performance status was a consistent prognostic factor in multivariate analysis. Other parameters such as serum LDH, CRP, and the REC showed a significant association neither with OS nor with the treatment response when they were considered as single factors. However, when integrated into a prognostic score, they were useful for risk stratification and discriminated groups with distinct survival probabilities. Thus, the risk score identified previously in a distinct 


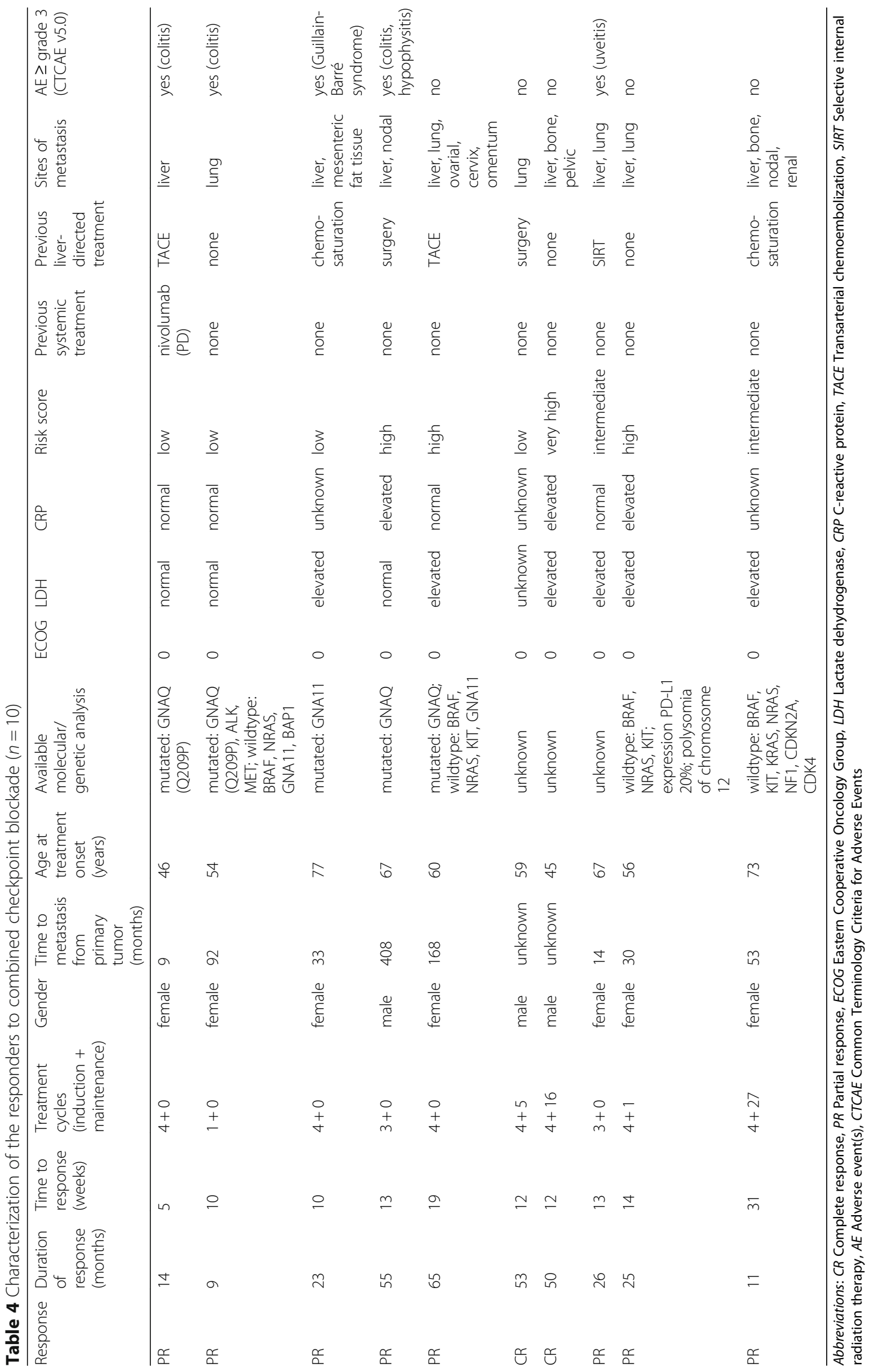

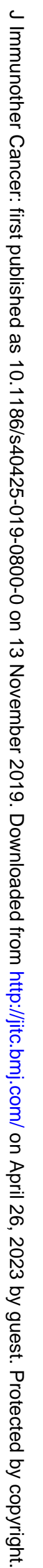


cohort was successfully validated in this population [6]. As there was a significant association neither with the ORR nor the DCR, we conclude that the score is generally prognostic but not specifically predictive for the response to checkpoint blockade.

The major limitations of this study are its retrospective design and the lack of a control group. When compared to historical controls, the median OS of 16.1 months is superior to survival estimates from other studies. Recently, the median OS benchmark for metastatic UM was identified as 10.2 months in a meta-analysis on individual data from 912 patients pooled from 29 trials [31]. Another analysis on individual-level data from 2494 patients proposed a median OS of 1.07 years across all treatment modalities. In this context, the OS observed in our cohort treated with combined checkpoint blockade appears more favorable, although external cohorts should be interpreted with caution and the comparison may be subject to significant confounding. A further limitation comes from the paucity of molecular and genetic analysis on the primary and metastatic tumors which are urgently needed to better characterize and understand the pattern of treatment response in UM.

\section{Conclusions}

Altogether, our study implies that combined checkpoint blockade represents the hitherto most effective treatment option available for metastatic UM available in routine care outside of clinical trials. Based on our analysis and preliminary data from others, we hypothesize that the ORR achieved with combined checkpoint blockade will be $15-17 \%$. Future trials are warranted to identify specific biomarkers for treatment response.

\section{Supplementary information}

Supplementary information accompanies this paper at https://doi.org/10. 1186/s40425-019-0800-0.

Additional file 1. Adverse events of combined checkpoint blockade according to frequency.

Additional file 2. Univariate Cox regression analysis of clinical and laboratory parameters.

\section{Abbreviations}

AE: Adverse event; Cl: Confidence interval; CRP: C-reactive protein; CTCAE: Common Terminology Criteria for Adverse Events; DCR: Disease control rate; ECOG: Eastern Cooperative Oncology Group; HR: Hazard ratio; LDH: Lactate dehydrogenase; ORR: Overall response rate; OS: Overall survival; PFS: Progression-free survival; Q2W: Every two weeks; Q3W: Every three weeks; REC: Relative eosinophil count; RLC: Relative lymphocyte count; RNC: Relative neutrophil count; UM: Uveal melanoma

\section{Acknowledgements}

Not applicable.

\section{Authors' contributions}

All authors made substantial contributions to the manuscript. MVH, MS and $\mathrm{CB}$ designed the study. MVH, TA, KCK, LH, JCH, MM, NK, CL, LR, JU, ED, AG,
CP, PT, K-MT, LZ, TKE, MCK, HMS and FM were involved in the acquisition of the dataset and interpreted the data. MVH and CB drafted the manuscript. All authors read and approved the final manuscript.

\section{Funding}

Not applicable.

\section{Availability of data and materials}

The datasets analysed during the current study are available from the corresponding author on reasonable request.

\section{Ethics approval and consent to participate}

This study was approved by the institutional review board of the medical faculty of the Munich University Hospital (approval number 413-16 UE) and was conducted in accordance with the principles of the Helsinki Declaration in its current version.

\section{Consent for publication}

Not applicable.

\section{Competing interests}

Teresa Amaral: grants from Neracare; travel support from Novartis, personal fees and travel support from BMS, outside the submitted work; Carola Berking: speaker's honoraria from BMS, Immunocore, MSD, Novartis, and Roche, consultant's honoraria from Amgen, BMS, MSD, Novartis, Pierre Fabre, Roche, and Sanofi-Aventis and travel support from Amgen, BMS, MSD, and Roche; Anja Gesierich: speaker's honoraria from Bristol-Myers Squibb, MSD Sharp \& Dohme and Roche, consultant's honoraria from Amgen, BristolMyers Squibb, Novartis, MSD Sharp \& Dohme, Pierre Fabre Pharmaceuticals, Pfizer, Roche and Sanofi Genzyme, travel support from Bristol-Myers Squibb, MSD Sharp \& Dohme, Novartis and Roche; Lucie Heinzerling speaker's/ consultant's honoraria from BMS, MSD, Novartis, Roche, Amgen, Pierre Fabre, Sanofi-Aventis, Curevac, research grants to institution from Novartis; Markus V. Heppt: speaker's honoraria and/or consultant's honoraria from Roche, Novartis, BMS, MSD and travel support from BMS; Katharina C. Kähler: consultant to Roche, BMS, MSD and received travel grants and speaker fees from Roche, BMS, MSD, Novartis, Amgen; Carmen Loquai: advisory for Roche, Amgen, Novartis, BMS, MSD, Ribological, speaker's honoraria from Roche, BMS, MSD, Novartis, travel reimbursement from Roche, BMS, MSD, Novartis; Max Schlaak: speaker's honoraria and/ or consultant's honoraria from Roche, Novartis, BMS, MSD, Kyowa Kirin, Amgen, Pierre Fabre and travel support from BMS; Henner M. Stege: travel support from Novartis and Roche; Patrick Terheyden: speaker's honoraria from BMS, Novartis, MSD, Pierre-Fabre, Curevac and Roche, consultant's honoraria from BMS, Novartis, Pierre-Fabre, Merck Serono, Sanofi und Roche and travel support fom BMS, Pierre-Fabre and Roche; Kai-Martin Thoms: speaker's honoraria from BMS, MSD, Novartis and Roche, consultant's honoraria from BMS, MSD, Novartis, Roche and Pierre Fabre and travel support from BMS, Novartis, Roche and Pierre Fabre; Jochen Utikal: advisory board or honoraria and travel support from Amgen, BMS, GSK, LeoPharma, MSD, Novartis, Pierre Fabre, Roche, outside the submitted work; Lisa Zimmer: consultant/ honoraria from Roche, BMS, MSD, Novartis, Sanofi, Pierre Fabre and travel support from MSD, BMS, Amgen, Pierre Fabre, Sanofi and Novartis. The remaining authors declare no conflict of interest.

\section{Author details}

'Department of Dermatology and Allergy, Munich University Hospital (LMU), Frauenlobstr. 9-11, 80337 Munich, Germany. ${ }^{2}$ Department of Dermatology, University Hospital Erlangen, Friedrich-Alexander-University Erlangen-Nürnberg (FAU), Ulmenweg 18, 91054 Erlangen, Germany. ${ }^{3}$ Department of Dermatology, Center for Dermatooncology, University Hospital Tübingen, Liebermeisterstr. 25, 72076 Tübingen, Germany.

${ }^{4}$ Portuguese Air Force Health Care Direction, Lisbon, Portugal. ${ }^{5}$ Department of Dermatology, University Hospital Schleswig-Holstein, Campus Kiel, Rosalind-Franklin-Str. 7, 24105 Kiel, Germany. ${ }^{6}$ Skin Cancer Center, Department of Dermatology and National Center for Tumor Diseases (NCT), University Hospital Heidelberg, Im Neuenheimer Feld 460, 69120 Heidelberg, Germany. ${ }^{7}$ Department of Dermatology, Venereology and Allergology, Goethe University, Theodor-Stern Kai 7, 60590 Frankfurt am Main, Germany. ${ }^{8}$ Department of Dermatology and Venereology, Skin Cancer Center at the Center of Integrated Oncology (ClO) Köln Bonn, University Hospital of Cologne, Kerpenerstr. 62, 50937 Cologne, Germany. ${ }^{9}$ Department of 
Dermatology, University Medical Center Mainz, Langenbeckstr. 1, 55131 Mainz, Germany. ${ }^{10}$ Department of Dermatology, Skin Cancer Center, Medical Faculty and University Hospital Carl Gustav Carus, TU Dresden, Fetscherstr. 74, 01307 Dresden, Germany. ${ }^{11}$ Skin Cancer Unit, German Cancer Research Center (DKFZ) and Department of Dermatology, Venereology and Allergology, University Medical Center Mannheim, Ruprecht-Karl University of Heidelberg, Theodor-Kutzer-Ufer 1-3, 68167 Mannheim, Germany.

${ }^{12}$ Department of Dermatology, Klinikum Ludwigshafen, Bremserstr. 79, 67063 Ludwigshafen, Germany. ${ }^{13}$ Department of Dermatology, University Hospital Würzburg, Josef-Schneider Straße 2, 97080 Würzburg, Germany.

${ }^{14}$ Department of Dermatology, Saarland University Medical School, Kirrbergerstr, 66421 Homburg/Saar, Germany. ${ }^{15}$ Department of Dermatology, University of Lübeck, Ratzeburger Allee 160, 23538 Lübeck, Germany. ${ }^{16}$ Department of Dermatology, University Medical Center Göttingen, Robert-Koch-Str. 40, 37075 Göttingen, Germany. ${ }^{17}$ Department of Dermatology, University Hospital, University Duisburg-Essen, Hufelandstr. 55, 45147 Essen, Germany.

Received: 20 July 2019 Accepted: 30 October 2019

\section{Published online: 13 November 2019}

\section{References}

1. Mallone S, De Vries E, Guzzo M, Midena E, Verne J, Coebergh JW, MarcosGragera R, Ardanaz E, Martinez R, Chirlaque MD, et al. Descriptive epidemiology of malignant mucosal and uveal melanomas and adnexal skin carcinomas in Europe. Eur J Cancer. 2012;48(8):1167-75.

2. Doherty RE, Alfawaz M, Francis J, Lijka-Jones B, Sisley K. Genetics of Uveal Melanoma. In: Scott JF, Gerstenblith MR, editors. Noncutaneous Melanoma. Brisbane: Codon Publications; 2018.

3. Rantala ES, Hernberg M, Kivela TT. Overall survival after treatment for metastatic uveal melanoma: a systematic review and meta-analysis. Melanoma Res. 2019;29(6):561-8.

4. Steeb T, Wessely A, Ruzicka T, Heppt MV, Berking C. How to MEK the best of uveal melanoma: a systematic review on the efficacy and safety of MEK inhibitors in metastatic or unresectable uveal melanoma. Eur J Cancer. 2018;103:41-51.

5. Heppt MV, Steeb T, Schlager JG, Rosumeck S, Dressler C, Ruzicka T, Nast A, Berking C. Immune checkpoint blockade for unresectable or metastatic uveal melanoma: a systematic review. Cancer Treat Rev. 2017;60:44-52.

6. Heppt MV, Heinzerling L, Kahler KC, Forschner A, Kirchberger MC, Loquai C, Meissner M, Meier F, Terheyden P, Schell B, et al. Prognostic factors and outcomes in metastatic uveal melanoma treated with programmed cell death-1 or combined PD-1/cytotoxic T-lymphocyte antigen-4 inhibition. Eur J Cancer. 2017:82:56-65.

7. Kottschade LA, McWilliams RR, Markovic SN, Block MS, Villasboas Bisneto J, Pham AQ, Esplin BL, Dronca RS. The use of pembrolizumab for the treatment of metastatic uveal melanoma. Melanoma Res. 2016;26(3):300-3.

8. Algazi AP, Tsai KK, Shoushtari AN, Munhoz RR, Eroglu Z, Piulats JM, Ott PA, Johnson DB, Hwang J, Daud Al, et al. Clinical outcomes in metastatic uveal melanoma treated with PD-1 and PD-L1 antibodies. Cancer. 2016;122(21):3344-53.

9. Zimmer L, Eigentler TK, Kiecker F, Simon J, Utikal J, Mohr P, Berking C, Kampgen E, Dippel E, Stadler R, et al. Open-label, multicenter, single-arm phase II DeCOG-study of ipilimumab in pretreated patients with different subtypes of metastatic melanoma. J Transl Med. 2015;13:351.

10. Larkin J, Chiarion-Sileni V, Gonzalez R, Grob JJ, Cowey CL, Lao CD, Schadendorf D, Dummer R, Smylie M, Rutkowski P, et al. Combined Nivolumab and Ipilimumab or Monotherapy in untreated melanoma. N Engl J Med. 2015;373(1):23-34.

11. Karivedu V, Eldessouki I, Taftaf A, Zhu Z, Makramalla A, Karim NA. Nivolumab and Ipilimumab in the treatment of metastatic Uveal melanoma: a singlecenter experience. Case Rep Oncol Med. 2019;2019:3560640.

12. Kirchberger MC, Moreira A, Erdmann M, Schuler G, Heinzerling L. Real world experience in low-dose ipilimumab in combination with PD-1 blockade in advanced melanoma patients. Oncotarget. 2018;9(48):28903-9.

13. Weide B, Martens A, Hassel JC, Berking C, Postow MA, Bisschop K, Simeone E, Mangana J, Schilling B, Di Giacomo AM, et al. Baseline biomarkers for outcome of melanoma patients treated with pembrolizumab. Clin Cancer Res. 2016;22(22):5487-96.

14. Martens A, Wistuba-Hamprecht K, Geukes Foppen M, Yuan J, Postow MA, Wong P, Romano E, Khammari A, Dreno B, Capone M, et al. Baseline peripheral blood biomarkers associated with clinical outcome of advanced melanoma patients treated with Ipilimumab. Clin Cancer Res. 2016;22(12):2908-18.
15. Hassel JC, Heinzerling L, Aberle J, Bahr O, Eigentler TK, Grimm MO, Grunwald V, Leipe J, Reinmuth N, Tietze JK, et al. Combined immune checkpoint blockade (anti-PD-1/anti-CTLA-4): evaluation and management of adverse drug reactions. Cancer Treat Rev. 2017;57:36-49.

16. Eigentler TK, Hassel JC, Berking C, Aberle J, Bachmann O, Grunwald V, Kahler KC, Loquai C, Reinmuth N, Steins M, et al. Diagnosis, monitoring and management of immune-related adverse drug reactions of anti-PD-1 antibody therapy. Cancer Treat Rev. 2016;45:7-18.

17. Eisenhauer EA, Therasse P, Bogaerts J, Schwartz LH, Sargent D, Ford R, Dancey J, Arbuck S, Gwyther S, Mooney M, et al. New response evaluation criteria in solid tumours: revised RECIST guideline (version 1.1). Eur J Cancer. 2009:45(2):228-47.

18. Rodrigues M, Mobuchon L, Houy A, Alsafadi S, Baulande S, Mariani O, Marande B, Ait Rais K, Van der Kooij MK, Kapiteijn E, et al. Evolutionary routes in metastatic Uveal melanomas depend on MBD4 alterations. Clin Cancer Res. 2019;25(18):5513-24.

19. Rodrigues M, Mobuchon L, Houy A, Fievet A, Gardrat S, Barnhill RL, Popova $T$, Servois $\mathrm{V}$, Rampanou A, Mouton A, et al. Outlier response to anti-PD1 in uveal melanoma reveals germline MBD4 mutations in hypermutated tumors. Nat Commun. 2018:9(1):1866.

20. Najjar Y, Navrazhina K, Bhatia R, Ding F, Abbate K, Durden B, Eroglu Z, Chowdhary A, Chandra S, Kennedy J, et al. Outcomes for patients with metastatic uveal melanoma (MUM) treated with ipilimumab and nivolumab (cIN): a multi-center, retrospective study. Pigment Cell Melanoma Res. 2019;32:92-172.

21. Piulats Rodriguez JM, De La Cruz ML, Espinosa E, Alonso Carrión L, Martin Algarra S, López-Castro R, Curiel García MT, Rodriguez Abreu D, Rullan Iriarte AJ, Berrocal JA. 1247PDPhase II multicenter, single arm, open label study of nivolumab in combination with ipilimumab in untreated patients with metastatic uveal melanoma (GEM1402.NCT02626962). Ann Oncol. 2018;29(suppl_8):mdy289.003.

22. Pelster M, Gruschkus SK, Bassett R, Gombos DS, Shephard M, Posada L, Glover M, Diab A, Hwu P, Patel SP. Phase II study of ipilimumab and nivolumab (ipi/nivo) in metastatic uveal melanoma (UM). J Clin Oncol. 2019;37(15):supplabstr 9522.

23. Karydis I, Chan PY, Wheater M, Arriola E, Szlosarek PW, Ottensmeier CH. Clinical activity and safety of Pembrolizumab in Ipilimumab pre-treated patients with uveal melanoma. Oncoimmunology. 2016;5(5):e1143997.

24. Rossi E, Pagliara MM, Orteschi D, Dosa T, Sammarco MG, Caputo CG, Petrone G, Rindi G, Zollino M, Blasi MA, et al. Pembrolizumab as first-line treatment for metastatic uveal melanoma. Cancer Immunol Immunother. 2019;68(7):1179-85.

25. Namikawa K, Takahashi A, Mori T, Tsutsumida A, Suzuki S, Motoi N, Jinnai S, Kage Y, Mizuta H, Muto Y, et al. Nivolumab for patients with metastatic uveal melanoma previously untreated with ipilimumab: a single-institution retrospective study. Melanoma Res. 2019. epub ahead of print.

26. van der Kooij MK, Joosse A, Speetjens FM, Hospers GA, Bisschop C, de Groot JW, Koornstra R, Blank CU, Kapiteijn E. Anti-PD1 treatment in metastatic uveal melanoma in the Netherlands. Acta Oncol. 2017;56(1):101-3.

27. Lawrence MS, Stojanov P, Polak P, Kryukov GV, Cibulskis K, Sivachenko A, Carter SL, Stewart C, Mermel CH, Roberts SA, et al. Mutational heterogeneity in cancer and the search for new cancer-associated genes. Nature. 2013;499(7457):214-8.

28. de Lange MJ, Nell RJ, Lalai RN, Versluis M, Jordanova ES, Luyten GPM, Jager MJ, van der Burg SH, Zoutman WH, van Hall T, et al. Digital PCR-based T-cell quantification-assisted Deconvolution of the microenvironment reveals that activated macrophages drive tumor inflammation in Uveal melanoma. Mol Cancer Res. 2018;16(12):1902-11.

29. Gezgin G, Dogrusoz M, van Essen TH, Kroes WGM, Luyten GPM, van der Velden PA, Walter V, Verdijk RM, van Hall T, van der Burg SH, et al. Genetic evolution of uveal melanoma guides the development of an inflammatory microenvironment. Cancer Immunol Immunother. 2017;66(7):903-12.

30. Hodi FS, Chiarion-Sileni V, Gonzalez R, Grob JJ, Rutkowski P, Cowey CL, Lao CD, Schadendorf D, Wagstaff J, Dummer R, et al. Nivolumab plus ipilimumab or nivolumab alone versus ipilimumab alone in advanced melanoma (CheckMate 067): 4-year outcomes of a multicentre, randomised, phase 3 trial. Lancet Oncol. 2018;19(11):1480-92.

31. Khoja L, Atenafu EG, Suciu S, Leyraz S, Sato T, Marshall E, Keilholz U, Zimmer L, Patel SP, Piperno-Neumann S, et al. Meta-analysis in metastatic Uveal melanoma to determine progression-free and overall survival benchmarks: an international rare cancers initiative (IRCI) ocular melanoma study. Ann Oncol. 2019. epub ahead of print.

\section{Publisher's Note}

Springer Nature remains neutral with regard to jurisdictional claims in published maps and institutional affiliations. 